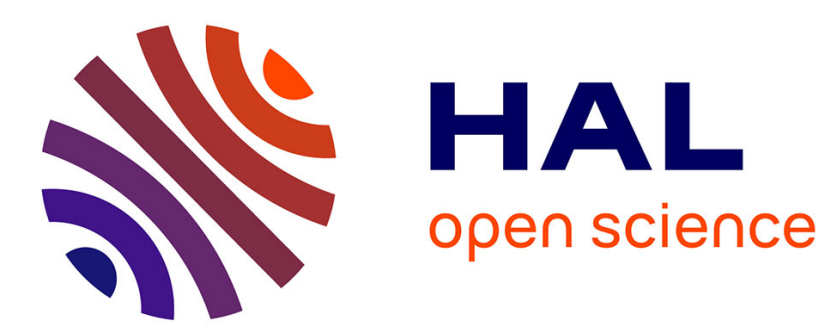

\title{
Convex conditions on decentralized control for graph topology preservation
}

Mirko Fiacchini, Irinel-Constantin Morarescu

\section{To cite this version:}

Mirko Fiacchini, Irinel-Constantin Morarescu. Convex conditions on decentralized control for graph topology preservation. IEEE Transactions on Automatic Control, 2014, 59 (6), pp.1640-1645. 10.1109/TAC.2013.2292719 . hal-00920914

\section{HAL Id: hal-00920914}

\section{https://hal.science/hal-00920914}

Submitted on 19 Dec 2013

HAL is a multi-disciplinary open access archive for the deposit and dissemination of scientific research documents, whether they are published or not. The documents may come from teaching and research institutions in France or abroad, or from public or private research centers.
L'archive ouverte pluridisciplinaire HAL, est destinée au dépôt et à la diffusion de documents scientifiques de niveau recherche, publiés ou non, émanant des établissements d'enseignement et de recherche français ou étrangers, des laboratoires publics ou privés. 


\title{
Convex conditions on decentralized control for graph topology preservation
}

\author{
Mirko Fiacchini*, Irinel-Constantin Morărescu\#
}

\begin{abstract}
The paper focuses on the preservation of a given graph topology which is usually chosen to ensure its connectivity. This is an essential ingredient allowing interconnected systems to accomplish tasks by using decentralized control strategies. We consider a networked system with discrete-time dynamics in which the subsystems are able to communicate if an algebraic relation between their states is satisfied. Each subsystem is called agent and the connected subsystems are called neighbors. The agents update their state in a decentralized manner by taking into account the neighbors' states. The characterization of the local control feedback gains ensuring topology preservation is provided. The results are based on invariance and set-theory and yield to conditions in Linear Matrix Inequality (LMI) form. The conditions for topology preservation are applied to an illustrative example concerning partial state consensus of agents with double integrator dynamics.
\end{abstract}

Index Terms-Interconnected systems, set-theory, consensus, decentralized control.

\section{INTRODUCTION}

Multi-agent systems have been used in the last decade to model different dynamics occurring in a large panel of applications going from biology and medicine to transportation, communication and sociology. It has also been emphasized that controlling interconnected systems in a decentralized manner [8], [10], [11] has advantages related to the computation and communication cost reduction. On the other hand the changes of the network topology may hamper the global coordination goal. To avoid this, recent works have been oriented towards the connectivity preservation of the interconnection graph of mobile networks [5], [13]. In [9] the authors compute a robust connected spanning subgraph which allows the highest degree of freedom for the agents position and find the initial states (position and velocities) assuring the graph preservation. Starting from this idea, in this paper we present conditions for network topology preservation. Note that the procedure may be designed for dynamic graph topologies with the intersection given by the core graph to be preserved.

The main contribution of this paper is the characterization of the control laws preserving a given graph. As in [13], the primary aim of our method is not a given global coordination objective. However, the controllers that pursue secondary global objectives, as flocking or rendez-vous, can be selected among those ensuring the graph topology preservation.

* GIPSA-lab, Grenoble Campus, 11 rue des Mathématiques, BP 46, 38402 Saint Martin d'Hères Cedex, France mirko.fiacchini@gipsa-lab. fr

\# Université de Lorraine, CRAN, UMR 7039, and CNRS, CRAN, UMR 7039, 2, Avenue de la Forêt de Haye, 54500, Vandœuvre, France constantin.moraresculuniv-lorraine.fr

This work has been partially founded by the Lorraine region's grant: "Analyse et conception de lois de commande pour des systèmes interconnectés".
We consider a networked system with discrete dynamics and a given interconnection topology. The subsystems are able to communicate if an algebraic relation, defined in the next section, between their states is satisfied. The connected subsystems are called neighbors. The subsystems update their state in a decentralized manner by taking into account their neighbors states. Each connection is preserved as far as the algebraic relation is verified. Our aim is to characterize the decentralized control laws that ensure the satisfaction of the algebraic constraint. The design of the decentralized controllers satisfying the algebraic constraint can be done either by minimizing a cost function [7], or by negociations through the network at each step [6]. Our approach use invariance based techniques (see [1]-[3] for the use of invariance in control theory) to characterize the conditions assuring that the algebraic constraint holds. The resulting topology preservation conditions rewrites as a convex constraint that may be posed in LMI form. Thus, we not only propose a new tool for decentralized control but also an easy implementable one.

\section{Notation}

The set of positive integers smaller than or equal to the integer $n \in \mathbb{N}$ is denoted as $\mathbb{N}_{n}$, i.e. $\mathbb{N}_{n}=\{x \in \mathbb{N}: 1 \leq$ $x \leq n\}$. Given the finite set $\mathscr{A} \subseteq \mathbb{N}_{n},|\mathscr{A}|$ is its cardinality. Given a symmetric matrix $P \in \mathbb{R}^{n \times n}$, notation $P>0(P \geq 0)$ means that $P$ is positive (semi-)definite. By $A^{\dagger}$ we denote the left pseudoinverse of the matrix $A$. Given the matrix $T \in \mathbb{R}^{n \times m}$ and $N \in \mathbb{N}$, $\operatorname{diag}_{N}(T) \in \mathbb{R}^{n N \times m N}$ is the blockdiagonal matrix whose $N$ block-diagonal elements are given by $T$, while $\operatorname{diag}(A, B, \ldots, Z)$ is the block-diagonal matrix, of adequate dimension, whose block-diagonal elements are the matrices $A, B, \ldots, Z$. Given a set of $N$ matrices $A_{k}$ with $k \in \mathbb{N}_{N}$, denote by $\left\{A_{k}\right\}_{k \in \mathbb{N}_{N}}$ the matrix obtained concatenating $A_{k}$ in column. Given a square matrix $A, \lambda_{\max }(A)$ denote the maximal eigenvalue of $A$.

\section{Problem statement}

Throughout the paper we consider a system consisting of $V \geq 2$ interconnected subsystems assumed identical, whose states have dimension $n \in \mathbb{N}$. Each subsystem is referred to as agent. Let us suppose that the initial interconnection topology is given by the graph $G=(\mathscr{V}, \mathscr{E})$ where the vertex set is $\mathscr{V}=$ $\mathbb{N}_{V}$ and the connecting edge set $\mathscr{E} \subseteq \mathscr{V} \times \mathscr{V}$ represents the set of pairs of agents that satisfy a distance-like condition. Precisely, for given $r>0, d \in \mathbb{N}, d \leq n$ and $T \in \mathbb{R}^{d \times n}$ with $T T^{\top}$ invertible, the initial edge set is given by

$$
\mathscr{E}=\left\{(i, j) \in \mathbb{N}_{V} \times \mathbb{N}_{V} \mid\left\|T\left(x_{i}(0)-x_{j}(0)\right)\right\|_{2}<r\right\},
$$


where $x_{i}$ and $x_{j}$ are the states of the $i$-th and $j$-th agents, respectively. Since $T$ is a matrix, i.e. a linear application, the graph $G$ is undirected, which means that $(i, j) \in \mathscr{E} \Leftrightarrow(j, i) \in \mathscr{E}$.

In the sequel we denote by $\mathscr{N} \subseteq \mathscr{E}$ the set of edges that must be preserved. Hence, it is sufficient to suppose that every agent $i$ knows the state of the $j$-th one if $(i, j) \in \mathscr{N}$.

Definition 1: For all $i \in \mathscr{V}$ we define the set of connected neighbors of the $i$-th agent as

$$
\mathscr{N}_{i}=\left\{j \in \mathbb{N}_{V}:(i, j) \in \mathscr{N}\right\} .
$$

The dynamics of each agent is given by

$$
x_{i}^{+}=A x_{i}+B u_{i},
$$

for all $i \in \mathbb{N}_{V}$, with $A \in \mathbb{R}^{n \times n}, B \in \mathbb{R}^{n \times m}$ and where $x_{i} \in \mathbb{R}^{n}$ is the state and $u_{i} \in \mathbb{R}^{m}$ is the control input of the $i$-th agent. As usual in multi-agent systems, the interconnection appears in the control input $u_{i}$ which is designed by taking into account not only the state $x_{i}$ but also the states $x_{j}$, for all $j \in \mathscr{N}_{i}$.

In order to clarify the concepts let us consider a network of mobile agents moving in a two dimensional space which are able to select the variation of their velocity. Modelling the input as a velocity variation or, equivalently, the variations along the two Cartesian axis, the dynamics of the $i$-th agent, with $i \in \mathbb{N}_{V}$, along the $x$ axis is given by

$$
\left\{\begin{array}{c}
p_{i}^{x}(k+1)=p_{i}^{x}(k)+t v_{i}^{x}(k), \\
v_{i}^{x}(k+1)=v_{i}^{x}(k)+u_{i}^{x}(k),
\end{array}\right.
$$

where $p_{i}^{x}$ is the position, $v_{i}^{x}$ the velocity, $u_{i}^{x}$ the control input and $t$ the sampling time. So, the overall dynamics of the $i$-th agent along the $x$ axis is given by a linear system with matrices

$$
\bar{A}=\left[\begin{array}{ll}
1 & t \\
0 & 1
\end{array}\right], \quad \bar{B}=\left[\begin{array}{l}
0 \\
1
\end{array}\right] .
$$

The dynamics along the $y$ axis are clearly analogous. Then the full dynamics of the $i$-th agent is given by (1) with

$$
A=\left[\begin{array}{cc}
\bar{A} & 0 \\
0 & \bar{A}
\end{array}\right], \quad B=\left[\begin{array}{cc}
\bar{B} & 0 \\
0 & \bar{B}
\end{array}\right],
$$

where the state is $x_{i}(k)=\left[p_{i}^{x}(k), v_{i}^{x}(k), p_{i}^{y}(k), v_{i}^{y}(k)\right]^{\top}$ and the input $u_{i}=\left[u_{i}^{x}, u_{i}^{y}\right]^{\top}$. Supposing that the agents can communicate only if the euclidean distance between them is smaller than $r$, is equivalent to define $T$ as the projection $T x_{i}(k)=$ $\left[p_{i}^{x}(k), p_{i}^{y}(k)\right]^{\top}$.

Given the set of connections $\mathscr{N}$, the objective is to design a decentralized control law ensuring that none of these connections is lost. Thus, the objective can be posed in terms of the error dynamics between connected neighbors:

$$
e_{i, j}^{+}=x_{i}^{+}-x_{j}^{+}=A\left(x_{i}-x_{j}\right)+B\left(u_{i}-u_{j}\right),
$$

for all $(i, j) \in \mathscr{N}$. In the sequel, the $i$-th input is defined by

$$
u_{i}=\sum_{j \in \mathscr{N}_{i}} K_{i, j}\left(x_{i}-x_{j}\right)=\sum_{j \in \mathscr{N}_{i}} K_{i, j} e_{i, j}
$$

with the controller gains $K_{i, j}$ depending on the current states and chosen such that the link $(i, j)$ is preserved. Thus, the design of each $u_{i}$ is reduced to the design of the controller gains associated to each $i, j$ system:

$$
e_{i, j}^{+}=\left(A+B K_{i, j}+B K_{j, i}\right) e_{i, j}+\sum_{k \in \mathscr{N}_{i}}^{k \neq j} B K_{i, k} e_{i, k}-\sum_{k \in \mathscr{N}_{j}}^{k \neq i} B K_{j, k} e_{j, k},
$$

for all $(i, j) \in \mathscr{N}$.

Remark 1: Our objective is to characterize the feedback gains involved in (5) such that the link $(i, j)$ is preserved. Since such a characterization must be shared by the $i$ and the $j$ agents to be useful in the decentralized context, only the information common to both of them should be involved. Such information consists in the states of the $i$ and $j$ agents and of those of the common neighbors. The shared information on the non-common neighbors reduces to their number (and implicitly a bound on their position). Such knowledge is used for determining a bound on the effect of the non-common neighbors on the $i, j$ system, as shown in the following.

The dynamics of the $i, j$ system is given by the matrix $A+B K_{i, j}+B K_{j, i}$ if no interaction with the other agents is present. The perturbation on the $i, j$ system induced by such an interaction can be bounded within a set depending on the radius $r$ and on the knowledge of common neighbors of the $i$-th and $j$-th agents. Consider the sets

$$
\begin{aligned}
& \mathscr{N}_{i, j}=\mathscr{N}_{i} \cap \mathscr{N}_{j}, \quad \overline{\mathscr{N}}_{i, j}=\mathscr{N}_{i} \backslash\left(\mathscr{N}_{i, j} \cup\{j\}\right), \\
& \overline{\mathscr{N}}_{j, i}=\mathscr{N}_{j} \backslash\left(\mathscr{N}_{i, j} \cup\{i\}\right),
\end{aligned}
$$

then, $\mathscr{N}_{i, j}$ denotes the common neighbors of the $i$-th and the $j$-th agents and $\overline{\mathscr{N}}_{i, j}$ the neighbors of the $i$-th one which are neither $j$ nor one of its neighbors, analogously for $\bar{N}_{j, i}$. The elements of $\bar{N}_{i, j} \cup \bar{N}_{j, i}$ are referred to as the non-common neighbors of the $i, j$ system. The dynamics of the $i, j$ system, perturbed by the non-common neighbors, is

$$
e_{i, j}^{+}=\left(A+B K_{i, j}+B K_{j, i}\right) e_{i, j}+\sum_{k \in \mathscr{N}_{i, j}}\left(B K_{i, k} e_{i, k}-B K_{j, k} e_{j, k}\right)+w_{i, j},
$$

with the bounded perturbation described by

$$
w_{i, j}=\sum_{k \in \bar{N}_{i, j}}\left(B K_{i, k} e_{i, k}\right)-\sum_{l \in \overline{\mathscr{N}}_{j, i}}\left(B K_{j, l} e_{j, l}\right) .
$$

For all the neighbors of the $j$-th and the $i$-th agents, the following relations hold

$$
\left\|T e_{i, k}\right\|_{2} \leq r, \quad \text { if } k \in \overline{\mathscr{N}}_{i, j}, \quad\left\|T e_{j, k}\right\|_{2} \leq r, \quad \text { if } k \in \overline{\mathscr{N}}_{j, i} .
$$

The problem addressed in this paper can be stated as follows.

Problem 1: Design a procedure to find at each step a condition on the decentralized control gains $K_{l, k}$, with $l, k \in \mathbb{N}_{V}$ in (7) and (8) such that the following algebraic relation is satisfied

$$
\left\|T e_{i, j}^{+}\right\|_{2}<r, \quad \forall(i, j) \in \mathscr{N} .
$$

\section{CONVEX CONDITIONS FOR TOPOLOGY PRESERVATION}

In the following, we restrict the study to the generic $i, j$ system with $(i, j) \in \mathscr{N}$. In this way, Problem 1 reduces to a set of simpler problems as explained below.

Problem 2: Given the $i, j$ system (7) and (8) with $(i, j) \in$ $\mathscr{N}$, pose a condition on the gains $K_{i, k}$, with $k \in \mathscr{N}_{i}$, and $K_{j, l}$, with $l \in \mathscr{N}_{j}$, such that the following algebraic relation is satisfied

$$
\left\|T e_{i, j}^{+}\right\|_{2}<r,
$$

if $\left\|T e_{i, k}\right\|_{2} \leq r,\left\|T e_{j, l}\right\|_{2} \leq r$ for all $e_{i, k}, k \in \mathscr{N}_{i}$ and $e_{j, l}, l \in \mathscr{N}_{j}$.

Let us consider the $2|\mathscr{N}|$-uples of gains $K_{i, j}, \quad(i, j) \in \mathscr{N}$ ordered lexicographically. We suppose that the Problem 2 is 
solved for the link $(i, j)$ and the solution is given by $S o l_{i j}$, the set of all tuples of gains satisfying its conditions. A solution of Problem 1 is then obtained by applying the following decentralized algorithm:

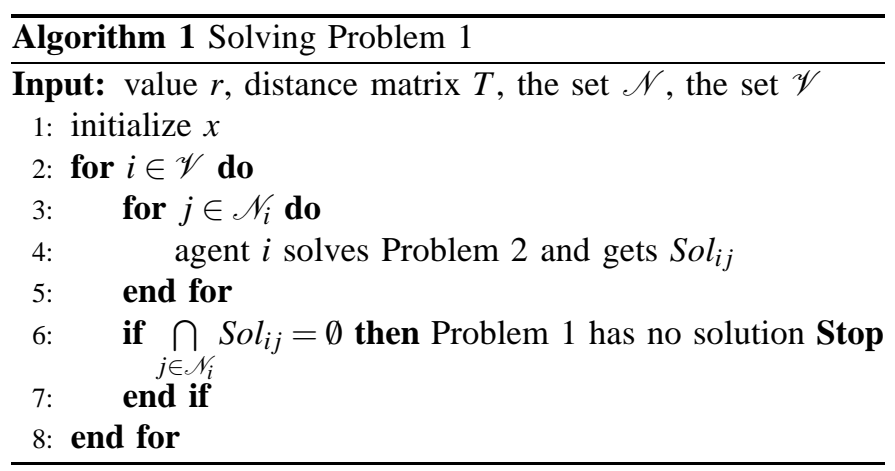

Remark 2: It is important to recall that in this paper we provide only necessary conditions and sufficient ones for the existence of controllers preserving the graph topology. Due to local knowledge it is clear that agent $i$ can only find conditions to preserve its own links. The design of the controllers preserving the whole topology can be done by minimizing cost functions that may be also related to a secondary agreement goal (see Section IV).

Definition 2: Given (6), (7) and (8), define

$$
\begin{array}{ll}
N=2\left|\mathscr{N}_{i, j}\right|+1, & \bar{N}=\left|\overline{\mathscr{N}}_{i, j}\right|+\left|\overline{\mathscr{N}}_{j, i}\right|, \\
E_{i, j}=\left\{e_{i, k}\right\}_{k \in \mathscr{N}_{i, j},}, & E_{j, i}=\left\{e_{j, k}\right\}_{k \in \mathscr{N}_{i, j},}, \\
E=\left[e_{i, j}^{\top}, E_{i, j}^{\top}, E_{j, i}^{\dagger}\right]^{\top} \in \mathbb{R}^{n N}, & Z=\operatorname{diag}_{N}(T) E \in \mathbb{R}^{d N}, \\
\check{K}_{i, j}=\left[\left\{K_{i, k}^{\top}\right\}_{k \in \mathscr{N}_{i, j}}\right]^{\top}, & \check{K}_{j, i}=\left[\left\{-K_{j, k}^{\top}\right\}_{k \in \mathscr{N}_{i, j}}\right]^{\top}, \\
\Delta=T\left[A+B\left(K_{i, j}+K_{j, i}\right),\right. & \left.B \check{K}_{i, j}, \quad B \check{K}_{j, i}\right] \operatorname{diag}_{N}(T)^{\dagger} \in \mathbb{R}^{d \times d N}, \\
\bar{E}_{i, j}=\left\{e_{i, k}\right\}_{k \in \overline{\mathscr{N}}_{i, j},} & \bar{E}_{j, i}=\left\{e_{j, k}\right\}_{k \in \overline{\mathscr{N}}_{j, i},}, \\
\bar{E}=\left[\bar{E}_{i, j}^{\top}, \bar{E}_{j, i}^{\top}\right]^{\top} \in \mathbb{R}^{n \bar{N}}, & \bar{Z}=\operatorname{diag}_{\bar{N}}(T) \bar{E} \in \mathbb{R}^{d \bar{N}}, \\
\hat{K}_{i, j}=\left[\left\{K_{i, k}^{\top}\right\}_{\left.k \in \overline{\mathscr{N}}_{\bar{i}, j}\right]^{\top},}\right. & \hat{K}_{j, i}=\left[\left\{-K_{j, k}^{\top}\right\}_{k \in \overline{\mathscr{N}}_{j, i}}\right]^{\top}, \\
\Gamma=T\left[B \hat{K}_{i, j}, B \hat{K}_{j, i}\right] \operatorname{diag}_{\bar{N}}(T)^{\dagger} \in \mathbb{R}^{d \times d \bar{N}} .
\end{array}
$$

Notice that $E, Z$ and $\Delta$ are two vectors and a matrix which are functions of the states and the gains of the $i, j$ system and its common neighbors; $\bar{E}, \bar{Z}$ and $\Gamma$ concern the states and gains of the non-common neighbors of the $i, j$ system. The dependence of $N, \bar{N}, E, Z, \Delta, \bar{E}, \bar{Z}$ and $\Gamma$ on the indices $i, j$ is omitted to simplify the notation. Using Definition 2, we have that $T e_{i, j}^{+}=\Delta Z+\Gamma \bar{Z}$ and then the algebraic constraint (11) is equivalent to

$$
[\Delta Z+\Gamma \bar{Z}]^{\top}[\Delta Z+\Gamma \bar{Z}]<r^{2},
$$

and the uncertainties bounds are given, in the terms of $\bar{Z}$, by

$$
\bar{Z}^{\top} D_{k} \bar{Z} \leq r^{2}, \quad \forall k \in \mathbb{N}_{\bar{N}},
$$

from (9), for all $k \in \mathbb{N}_{\bar{N}}$, with

$$
D_{k}=\operatorname{diag}\left(0_{d}, \ldots, 0_{d}, I_{d}, 0_{d}, \ldots, 0_{d}\right) \in \mathbb{R}^{d \bar{N} \times d \bar{N}},
$$

where $I_{d} \in \mathbb{R}^{d \times d}$ is the identity, $0_{d} \in \mathbb{R}^{d \times d}$ is the zero matrix and $k$ indicates that $I_{d}$ is the $k$-th block of $D_{k}$.

Remark 3: We have shown that Problem 2 is equivalent to: (13) is satisfied for every $\bar{Z} \in \mathbb{R}^{d \bar{N}}$ fulfilling (14).

\section{A. Necessary condition for network preservation}

In this section we derive some bounds on the gains associated to the non-common neighbors that are necessary for the feasibility of Problem 2. In other words, we look for constraints on the matrix $\Gamma$ that hampers the existence of admissible realizations of the uncertainty $\bar{Z}$ which lead to the break of the $(i, j)$ link.

Consider the matrix $\Gamma^{\top} \Gamma$ which is real, symmetric, positive semidefinite and block diagonal, i.e. there are $M_{k} \in \mathbb{R}^{d \times d}$, with $k \in \mathbb{N}_{\bar{N}}$, real, symmetric and positive semidefinite such that $\Gamma^{\top} \Gamma=\operatorname{diag}\left(M_{1}, \ldots, M_{\bar{N}}\right)$. Then there exist $G_{k} \in \mathbb{R}^{d \times d}$ diagonal and $H_{k} \in \mathbb{R}^{d \times d}$ unitary, for all $k \in \mathbb{N}_{\bar{N}}$, such that

$$
\begin{aligned}
\Gamma^{\top} \Gamma & =\operatorname{diag}\left(M_{1}, \ldots, M_{\bar{N}}\right) \\
& =\operatorname{diag}\left(H_{1}^{\top} G_{1} H_{1}, \ldots, H_{\bar{N}}^{\top} G_{\bar{N}} H_{\bar{N}}\right)=H^{\top} G H,
\end{aligned}
$$

with $G=\operatorname{diag}\left(G_{1}, \ldots, G_{\bar{N}}\right)$ and $H=\operatorname{diag}\left(H_{1}, \ldots, H_{\bar{N}}\right)$ such that $H H^{\top}=I_{d \bar{N}}$. The diagonal entries of $G_{k}$ (resp. of $G$ ) are the eigenvalues of $M_{k}$ (resp. of $\Gamma^{\top} \Gamma$ ) and the columns of $H_{k}^{\top}$ (resp. of $H^{\top}$ ) are the related eigenvectors, for all $k \in \mathbb{N}_{\bar{N}}$. All the eigenvalues are nonnegative.

Assumption 1: The matrix $\Gamma$ is such that $\sum_{k \in \mathbb{N}_{\bar{N}}} \lambda_{\max }\left(M_{k}\right)<1$. The necessity of Assumption 1 is proved below.

Proposition 1 (Necessary condition): If Assumption 1 does not hold, then there is $\bar{Z} \in \mathbb{R}^{d \bar{N}}$ such that (14) holds and

$$
[\Delta Z+\Gamma \bar{Z}]^{\top}[\Delta Z+\Gamma \bar{Z}] \geq r^{2},
$$

for all $Z \in \mathbb{R}^{d N}$ and $\Delta$.

Proof: Given $\Gamma^{\top} \Gamma$ as in (15), denote with $\lambda_{k}$ the maximal eigenvalue of $M_{k}$, i.e. $\lambda_{k}=\lambda_{\max }\left(M_{k}\right)$, and suppose with no loss of generality that $\lambda_{k}$ is the first element of the diagonal of $G_{k}$, for every $k \in \mathbb{N}_{\bar{N}}$. Defining with $h_{k} \in \mathbb{R}^{d}$ an eigenvector of norm one related to $\lambda_{k}$ we have $H_{k} h_{k}=[1,0, \ldots, 0]^{\top}$, for all $k \in \mathbb{N}_{\bar{N}}$.

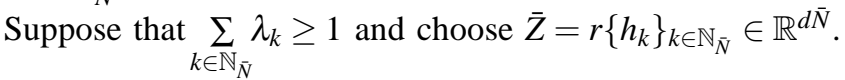
We have that

$$
\bar{Z}^{\top} D_{k} \bar{Z}=r^{2} h_{k}^{\top} h_{k}=r^{2},
$$

for all $k \in \mathbb{N}_{\bar{N}}$, thus $\bar{Z}$ fulfills (14). Moreover

$$
\begin{aligned}
& \bar{Z}^{\top} \Gamma^{\top} \Gamma \bar{Z}=\bar{Z}^{\top} H^{\top} G H \bar{Z}= \\
& r^{2} \sum_{k \in \mathbb{N}_{\bar{N}}}[1,0, \ldots, 0] G_{k}[1,0, \ldots, 0]^{\top}=r^{2} \sum_{k \in \mathbb{N}_{\bar{N}}} \lambda_{k} \geq r^{2} .
\end{aligned}
$$

Notice that (17) and (18) do not depend on the sign of $\bar{Z}$. Thus, for all $Z$ and $\Delta$ there exists an adequate selection of the sign of $\bar{Z}$ such that

$$
Z^{\top} \Delta^{\top} \Delta Z+2 \bar{Z}^{\top} \Gamma^{\top} \Delta Z+\bar{Z}^{\top} \Gamma^{\top} \Gamma \bar{Z} \geq \bar{Z}^{\top} \Gamma^{\top} \Gamma \bar{Z}
$$

Actually, the first term in (19) is always nonnegative and the second term in (19) becomes nonnegative by appropriately choosing the sign of $\bar{Z}$. From (18) and (19) we have (16).

An alternative necessary condition for the Problem 2 to have a solution, inspired by the comments of an anonymous reviewer, follows from the lemma below. This condition is employed in the next section to provide sufficient conditions for Problem 2 to admit solutions. 
Lemma 1: The matrix $\Gamma$ satisfies Assumption 1 if and only if there exists $\Lambda=\operatorname{diag}\left(\lambda_{1} I_{d}, \ldots, \lambda_{\bar{N}} I_{d}\right)$ with $\lambda_{k} \geq 0$ and $\sum_{k \in \mathbb{N}_{\bar{N}}} \lambda_{k}<1$ such that

$$
\Gamma^{\top} \Gamma<\Lambda
$$

Proof: For the necessity, suppose that Assumption 1 holds. Denote $\varepsilon=1-\sum_{k \in \mathbb{N}_{\bar{N}}} \lambda_{\max }\left(M_{k}\right)>0$ and define $\Lambda$ with $\lambda_{k}=$ $\lambda_{\max }\left(M_{k}\right)+0.5 \varepsilon / \bar{N}$. Clearly (20) holds and

$$
\sum_{k \in \mathbb{N}_{\bar{N}}} \lambda_{k}=\sum_{k \in \mathbb{N}_{\bar{N}}} \lambda_{\max }\left(M_{k}\right)+0.5 \varepsilon<\sum_{k \in \mathbb{N}_{\bar{N}}} \lambda_{\max }\left(M_{k}\right)+\varepsilon=1 .
$$

For the sufficiency, suppose the existence of $\Lambda$ that satifies (20) with $\sum_{k \in \mathbb{N}_{\bar{N}}} \lambda_{k}<1$. From the particular structure of $\Gamma$, (20) implies $M_{k}<\lambda_{k} I_{d}$ which yields $\lambda_{\max }\left(M_{k}\right)<\lambda_{k}$, for all $k \in \mathbb{N}_{\bar{N}}$. From this and $\sum_{k \in \mathbb{N}_{\bar{N}}} \lambda_{k}<1$, Assumption 1 follows.

Remark 4: Given $\Lambda$ as in Lemma 1 , the quantity $\delta=$ $\sum \lambda_{k}$ may be geometrically interpreted as a bound on the $k \in \mathbb{N}_{\bar{N}}$ uncertainty. In fact, from Definition 2, condition (20) leads to

$$
\left\|T w_{i, j}\right\|_{2}^{2}=\bar{Z}^{\top} \Gamma^{\top} \Gamma \bar{Z}<\bar{Z}^{\top} \Lambda \bar{Z}=\sum_{k \in \mathbb{N}_{\bar{N}}} \lambda_{k} \bar{Z}^{\top} D_{k} \bar{Z} \leq \sum_{k \in \mathbb{N}_{\bar{N}}} \lambda_{k} r^{2}
$$

for all $e_{i, k}$, with $k \in \overline{\mathscr{N}}_{i, j}$, and $e_{j, k}$ with $k \in \overline{\mathscr{N}}_{j, i}$ such that (9) holds. Precisely, the effect of the non-common neighbors can be modelled as a perturbation on the $i, j$ system bounded by an ellipsoid determined by $T^{\top} T$ and of radius $\sqrt{\delta} r$. Therefore the condition $\Gamma^{\top} \Gamma<\Lambda$ results in a bound on the gains related to the non-common neighbors of the $i$-th and $j$-th agents. It can be interpreted as a joint limitation on the control efforts of the agents $i$ and $j$ aimed at regulating and preserving their other connections.

Using the interpretation above, Proposition 1 may be reformulated as an intuitive result saying that: it is not possible to guarantee the "distance" constraint as far as the uncertainties are too large, i.e. $\left\|T w_{i, j}\right\|_{2} \geq r$.

\section{B. Sufficient condition for network preservation}

This section provides conditions on the gains $\Gamma$ and $\Lambda$ for guaranteeing the existence of solutions for Problem 2. In order to derive the sufficient condition we use the S-procedure (see [4] for further details). This commonly used procedure gives sufficient conditions in terms of LMI. An important contribution of this paper, namely the sufficient condition for the constraint (11) to hold, is stated in the following theorem.

Theorem 1 (Sufficient condition): Problem 2 admits solutions (i.e. condition (13) is satisfied for every $\bar{Z} \in \mathbb{R}^{d \bar{N}}$ fulfilling (14) ) if there exists $\Lambda=\operatorname{diag}\left(\lambda_{1} I_{d}, \ldots, \lambda_{\bar{N}} I_{d}\right)$ with $\lambda_{k} \geq 0$, for all $k \in \mathbb{N}_{\bar{N}}$ such that

$$
\left[\begin{array}{ccc}
r^{2}-r^{2} \delta & 0 & Z^{\top} \Delta^{\top} \\
0 & \Lambda & \Gamma^{\top} \\
\Delta Z & \Gamma & I_{d}
\end{array}\right]>0,
$$

with $\delta=\sum_{k \in \mathbb{N}_{\bar{N}}} \lambda_{k}$. Furthermore, any $(\Delta, \Gamma)$ satisfying (21) defines admissible controller gains for the Problem 2.
Proof: First notice that every solution of (21) satisfies also

$$
\sum_{k \in \mathbb{N}_{\bar{N}}} \lambda_{k}<1, \quad \Gamma^{\top} \Gamma-\Lambda<0
$$

as the principal minors of a positive definite matrix are positive definite. Since (22) is a necessary condition for the Problem 2 to admit a solution, see Section III-A, there is no loss of generality in assuming it satisfied. Condition (13) is equivalent to

$$
\left[Z^{\top}, \bar{Z}^{\top}\right]\left[\begin{array}{cc}
\Delta^{\top} \Delta & \Delta^{\top} \Gamma \\
\Gamma^{\top} \Delta & \Gamma^{\top} \Gamma
\end{array}\right]\left[\begin{array}{c}
Z \\
\bar{Z}
\end{array}\right]<r^{2} .
$$

This condition must be satisfied for every $\bar{Z}$ such that (14) holds. Applying the $S$-procedure, a sufficient condition for (13) to hold for every $\bar{Z} \in \mathbb{R}^{d \bar{N}}$ satisfying (14) is the existence of $\lambda_{k} \geq 0$, for all $k \in \mathbb{N}_{\bar{N}}$, such that

$$
Z^{\top} \Delta^{\top} \Delta Z+2 \bar{Z}^{\top} \Gamma^{\top} \Delta Z+\bar{Z}^{\top}\left[\Gamma^{\top} \Gamma-\Lambda\right] \bar{Z}<r^{2}-r^{2} \delta,
$$

for every $\bar{Z} \in \mathbb{R}^{d \bar{N}}$. From (22) and $Z$ being known, the lefthand side of (24) is a concave function in $\bar{Z}$ whose maximum is attained at

$$
\bar{Z}=-\left(\Gamma^{\top} \Gamma-\Lambda\right)^{-1} \Gamma^{\top} \Delta Z .
$$

Hence condition (24) holds for every $\bar{Z} \in \mathbb{R}^{d \bar{N}}$ if and only if it is satisfied for the maximum of the function at left-hand side, that is if and only if

$$
Z^{\top} \Delta^{\top} \Delta Z-Z^{\top} \Delta^{\top} \Gamma\left(\Gamma^{\top} \Gamma-\Lambda\right)^{-1} \Gamma^{\top} \Delta Z<r^{2}-r^{2} \delta,
$$

which is given by (24) at (25). Hence every $\Lambda, \Delta$ and $\Gamma$ satisfying conditions (22) and (26) ensure the satisfaction of $\left\|T e_{i, j}^{+}\right\|_{2}<r$ for all $\bar{Z}$ such that (14) holds. The condition (26) is equivalent to

$$
\begin{aligned}
& {\left[\begin{array}{cc}
Z^{\top} \Delta^{\top} \Delta Z-r^{2}+r^{2} \delta & Z^{\top} \Delta^{\top} \Gamma \\
\Gamma^{\top} \Delta Z & \Gamma^{\top} \Gamma-\Lambda
\end{array}\right]<0 } \\
\Leftrightarrow \quad & {\left[\begin{array}{cc}
Z^{\top} \Delta^{\top} \Delta Z & Z^{\top} \Delta^{\top} \Gamma \\
\Gamma^{\top} \Delta Z & \Gamma^{\top} \Gamma
\end{array}\right]<\left[\begin{array}{cc}
r^{2}-r^{2} \delta & 0 \\
0 & \Lambda
\end{array}\right] } \\
\Leftrightarrow \quad & {\left[\begin{array}{c}
Z^{\top} \Delta^{\top} \\
\Gamma^{\top}
\end{array}\right]\left[\begin{array}{cc}
\Delta Z & \Gamma
\end{array}\right]<\left[\begin{array}{cc}
r^{2}-r^{2} \delta & 0 \\
0 & \Lambda
\end{array}\right] } \\
\Leftrightarrow \quad & {\left[\begin{array}{ccc}
r^{2}-r^{2} \delta & 0 & Z^{\top} \Delta^{\top} \\
0 & \Lambda & \Gamma^{\top} \\
\Delta Z & \Gamma & I_{d}
\end{array}\right]>0 . }
\end{aligned}
$$

Thus (21) is equivalent to (24), sufficient condition for (13) to hold.

\section{Guaranteed network preservation: common feedback gains}

The condition presented in the previous subsection ensures that the algebraic constraint related to the $i, j$ system is satisfied at the successive time instant. No insurance on its satisfaction along the evolution of the overall system can be guaranteed, unless proper choices of $K_{i, j}$ are done. In case the feedback gains are assumed to be the same for every agent and every $i, j$ system, a sufficient condition for guaranteeing the network topology preservation at every future time instant can be posed.

Assumption 2: Given the system (1) with control (4), assume that $K_{i, j}=\bar{K}$ for all $(i, j) \in \mathscr{N}$. 
The objective is to characterize the set of common feedback gains such that, if applied to control the multi-agent system, they ensure the non-increasing of the values $\left\|T e_{i, j}\right\|_{2}$ for all $(i, j) \in \mathscr{N}$. If the connection condition is satisfied by the initial condition, i.e. $\left\|T e_{i, j}(0)\right\|_{2} \leq r$ for all $(i, j) \in \mathscr{N}$, the network topology preservation is iteratively guaranteed at every successive instant. Given the sets as in (6), define

$$
N_{M}=\max _{(i, j) \in \mathscr{N}}\left\{\left|\mathscr{N}_{i}\right|+\left|\mathscr{N}_{j}\right|-2\right\} .
$$

Then, for all $(i, j) \in \mathscr{N}, N_{M} \in \mathbb{N}$ is an upper bound of the number of agents different from $i$ and $j$ affecting the $i, j$ system.

Proposition 2: Let Assumption 2 hold. If there exists $\lambda \in$ $[0,1]$ such that

$$
\begin{gathered}
{\left[\begin{array}{cc}
\lambda T^{\top} T & (A+2 B \bar{K})^{\top} T^{\top} \\
T(A+2 B \bar{K}) & \lambda I_{d}
\end{array}\right] \geq 0,} \\
{\left[\begin{array}{cc}
(1-\lambda) T^{\top} T & N_{M} \bar{K}^{\top} B^{\top} T^{\top} \\
N_{M} T B \bar{K} & (1-\lambda) I_{d}
\end{array}\right] \geq 0,}
\end{gathered}
$$

then the systems given by (7) and (8) are such that $\left\|T e_{i, j}^{+}\right\|_{2} \leq r$ for all $(i, j) \in \mathscr{N}$ if $\left\|T e_{l, k}\right\|_{2} \leq r$ for all $(l, k) \in \mathscr{N}$.

Proof: Define the set $\mathscr{B}_{T}=\left\{e \in \mathbb{R}^{n}:\|T e\|_{2} \leq r\right\}$, then $e \in$ $\mathscr{B}_{T}$ if and only if $e^{\top} T^{\top} T e \leq r^{2}$. The first condition in (27) is equivalent to $(A+2 B \bar{K})^{\top} T^{\top} T(A+2 B \bar{K}) \leq \lambda^{2} T^{\top} T$, which implies that $(A+2 B \bar{K}) \mathscr{B}_{T} \subseteq \lambda \mathscr{B}_{T}$. From Assumption 2 one have that $K_{i, j}=K_{j, i}=\bar{K}$, which means that $A+2 B \bar{K}$ is the dynamics of any $i, j$ system in the absence of the perturbation of the neighbors. Then the set $\mathscr{B}_{T}$ is mapped in $\lambda \mathscr{B}_{T}$ if no perturbation is present, that is $\left(A+B K_{i, j}+B K_{j, i}\right) e_{i, j} \in \lambda \mathscr{B}_{T}$, for all $e_{i, j} \in \mathscr{B}_{T}$. Analogously, the second condition in (27) is equivalent to $N_{M}^{2} \bar{K}^{\top} B^{\top} T^{\top} T B \bar{K} \leq(1-\lambda)^{2} T^{\top} T$, which leads to $\sum_{k \in \mathbb{N}} B \bar{K} \mathscr{B}_{T}=N_{M} B \bar{K} \mathscr{B}_{T} \subseteq(1-\lambda) \mathscr{B}_{T}$. This means that if $k \in \mathbb{N}_{N_{M}}$

$e_{i, k} \in \mathscr{B}_{T}$ for all $k \in \mathscr{N}_{i} \backslash\{j\}$ and $e_{k, j} \in \mathscr{B}_{T}$ for all $k \in \mathscr{N}_{j} \backslash\{i\}$, as implicitly assumed, then

$\sum_{k \in \mathscr{N}_{i, j}}\left(B \bar{K} e_{i, k}-B \bar{K} e_{j, k}\right)+\sum_{k \in \mathscr{N}_{i, j}}\left(B \bar{K} e_{i, k}\right)-\sum_{l \in \bar{N}_{j, i}}\left(B \bar{K} e_{j, l}\right) \in(1-\lambda) \mathscr{B}_{T}$,

for all $(i, j) \in \mathscr{N}$. From properties of the Minkowski set addition, see [12], we have $e_{i, j}^{+} \in \lambda \mathscr{B}_{T}+(1-\lambda) \mathscr{B}_{T}=\mathscr{B}_{T}$, if $e_{l, k} \in \mathscr{B}_{T}$ for all $(l, k) \in \mathscr{N}$, which ends the proof.

Proposition 2 characterizes the common gains that ensure the network topology preservation along the whole trajectories. An analogous sufficient condition for convergence follows.

Corollary 1: Let Assumption 2 hold. If there exist $\lambda \in[0,1]$ and $\bar{\lambda}>0$ such that

$$
\begin{gathered}
{\left[\begin{array}{cc}
(\lambda-\bar{\lambda}) T^{\top} T & (A+2 B \bar{K})^{\top} T^{\top} \\
T(A+2 B \bar{K}) & (\lambda-\bar{\lambda}) I_{d}
\end{array}\right] \geq 0,} \\
{\left[\begin{array}{cc}
(1-\lambda) T^{\top} T & N_{M} \bar{K}^{\top} B^{\top} T^{\top} \\
N_{M} T B \bar{K} & (1-\lambda) I_{d}
\end{array}\right] \geq 0,}
\end{gathered}
$$

then the systems given by (7) and (8) are such that

$$
\left\|T e_{i, j}^{+}\right\|_{2} \leq(1-\bar{\lambda})\left\|T e_{i, j}\right\|_{2},
$$

for all $(i, j) \in \mathscr{N}$ if $e_{l, k} \in \mathbb{R}^{n}$ satisfies $\left\|T e_{l, k}\right\|_{2} \leq r$ for all $(l, k) \in \mathscr{N}$.

Hence the corollary provides a sufficient condition for the exponential convergence of the $i, j$ system to the set $\|T e\|_{2}=0$, for all $(i, j) \in \mathscr{N}$, if $\left\|T e_{l, k}(0)\right\|_{2} \leq r$ for every $(l, k) \in \mathscr{N}$. Notice that this would imply $\left\|T e_{i, j}^{+}\right\|_{2}<r$, strictly, as required in Problem 2.

Proposition 2 and Corollary 1 provide sufficient conditions on the local feedback gains for the recursive satisfaction of the algebraic constraints and for convergence, respectively. On the other hand, the price to pay for the recursive guarantee of constraints satisfaction (or of convergence) is a certain degree of conservativeness of the results in comparison with those of Theorem 1.

\section{ApPliCATION TO DECENTRALIZED CONTROL OF MULTI-AGENT SYSTEMS}

Let us consider the problem of flocking for a set of agents with the dynamics along the $x$ axis given by (2) and similar one along $y$ axis. We consider an interaction graph in which the euclidean distance between two neighbors is smaller than or equal to $r$. The matrix $T$ defining the algebraic constraint to be preserved is

$$
T=\left[\begin{array}{llll}
1 & t & 0 & 0 \\
0 & 0 & 1 & t
\end{array}\right] .
$$

Thus, denoting $\Delta p_{i, j}=\left(\left(p_{i}^{x}(k)-p_{j}^{x}(k)\right)^{2}+\left(p_{i}^{y}(k)-p_{j}^{y}(k)\right)^{2}\right)^{\frac{1}{2}}$ we suppose $\Delta p_{i, j}(k+1) \leq r$ and we determine the controllers ensuring $\Delta p_{i, j}(k+2) \leq r$. Among all these controllers we chose the one that minimize the difference between neighbors speeds, i.e. the value $\Delta v_{i, j}=\left(\left(v_{i}^{x}(k)-v_{j}^{x}(k)\right)^{2}+\left(v_{i}^{y}(k)-\right.\right.$ $\left.\left.v_{j}^{y}(k)\right)^{2}\right)^{\frac{1}{2}}$. Let us consider the six interconnected agents with the initial conditions given in [9] and connected by the minimal robust graph computed in the same work. That is: $\mathscr{N}=\{(1,2),(2,3),(3,4),(4,5),(5,6)\}, r=3.2$ and initial conditions:

$$
\begin{array}{lll}
x_{1}(0) & =\left[\begin{array}{llll}
-4 & -v_{0} & 3 & 0
\end{array}\right]^{\top}, & x_{6}(0)=\left[\begin{array}{llll}
4 & v_{0} & 3 & 0
\end{array}\right]^{\top}, \\
x_{2}(0)=\left[\begin{array}{llll}
-2 & -v_{0} & 2 & 0
\end{array}\right]^{\top}, & x_{5}(0)=\left[\begin{array}{llll}
2 & v_{0} & 2 & 0
\end{array}\right]^{\top}, \\
x_{3}(0)=\left[\begin{array}{llll}
-1 & -v_{0} & 0 & 0
\end{array}\right]^{\top}, & x_{4}(0)=\left[\begin{array}{llll}
1 & v_{0} & 0 & 0
\end{array}\right]^{\top},
\end{array}
$$

where $v_{0}$ is used as a parameter to analyze the maximal initial speed that can be dealt with by different control strategies. It is noteworthy that, as shown in [9], for the classical consensus algorithm the preservation of the minimal robust graph is guaranteed for a critical speed value $v_{c} \simeq 0.23$. Nevertheless, it is numerically shown that the sufficient condition is conservative since for $v_{0}=1.5 v_{c}$ (generating approximately a 4 times higher global velocity disagreement) the robust graph is not broken. We also note that the classical consensus algorithm is not able to preserve the connectivity when the global disagreement is 5 times superior to the one guaranteeing the consensus (i.e. $\left.v_{0}>2.1 v_{c}\right)$.

In the sequel, we use Theorem 1 to compute the sets of gains ensuring the topology preservation. The choice of the controllers gain within these sets is done heuristically by minimizing a cost function that expresses the velocity disagreement and penalizes the links break. This strategy has admissible solutions for $v_{0}=19 v_{c}$ (see Figures 1,2) and the connection between the third and the fourth agent is lost for $v_{0}=20 v_{c}$. It is worth noting that the control acts like springs between agents' velocities (compare the Figures 1 and 2, noticing that 
the system 1,2 has the same evolution as the 5,6 and the 2,3 the same as 4,5 , by symmetry). First, the control cancels the speed difference between neighbors with opposite velocities creating a speed disagreement in both symmetric branches of the graph. Next, it cancel the disagreement between 2-nd and the 3-rd agent and between the 4-th and 5-th one, mimicking a gossiping procedure where the choice of active communication link is given by the error between neighbors speeds. Doing so, either the flocking is reached before the connectivity is lost, or the graph splits into two groups that will independently agree to two different velocity values.
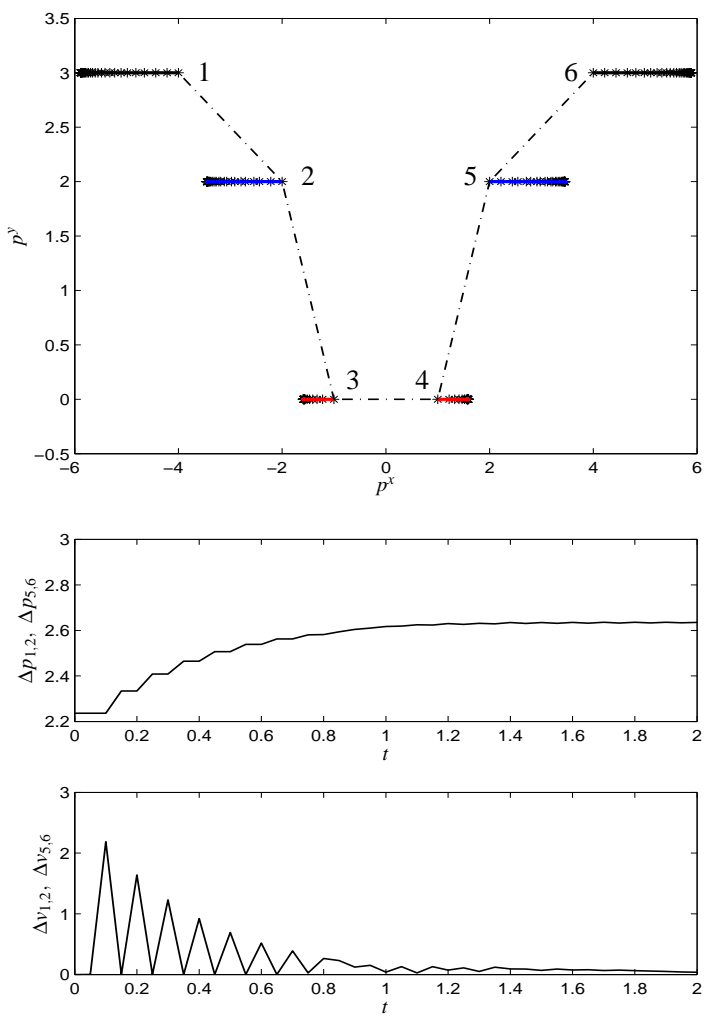

Fig. 1. Trajectories (top) and errors of the systems 1,2 and 5,6, (bottom).

\section{CONCLUSION AND FURTHER WORKS}

In this paper we have provided necessary conditions and sufficient ones in terms of controller gains for the preservation of a given graph topology. These conditions are written as a convex constraint that may be posed in LMI form allowing an easy implementation of the controller design. An illustrative example shows how the procedure can be applied to achieve both the topology preservation and an additional global objective which is in this case the partial state consensus. In this example our controller allows to solve the coordination problem for a set of initial conditions that is larger than the one existing in the literature.

\section{REFERENCES}

[1] G. Basile and G Marro. Controlled and Conditioned Invariants in Linear system Theory. Prentice Hall, 1992.

[2] D.P. Bertsekas. Infinite-time reachability of state-space regions by using feedback control. IEEE Transactions on Automatic Control, 17:604-613, 1972.
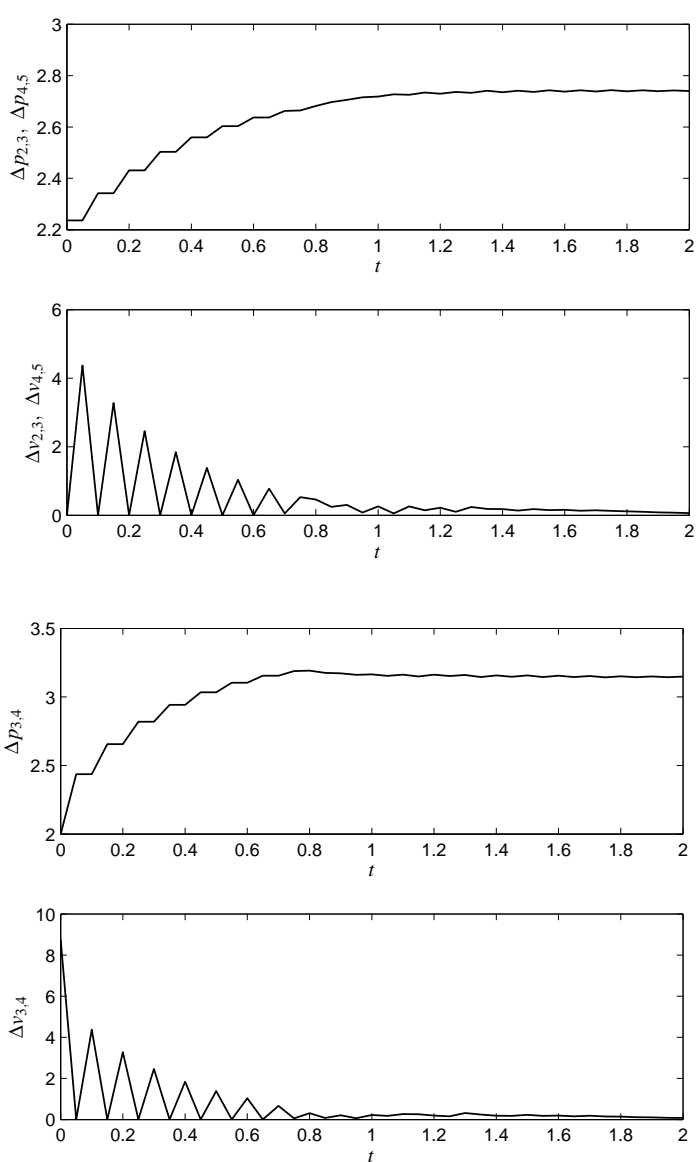

Fig. 2. Errors of the systems 2,3 and 4,5 (top) and the 3,4 one (bottom).

[3] F. Blanchini and S. Miani. Set-Theoretic Methods in Control. Birkhäuser, 2008.

[4] S. Boyd, L. El Ghaoui, E. Feron, and V. Balakrishnan. Linear Matrix Inequalities in system and control theory. SIAM, 1994.

[5] F. Bullo, J. Cortés, and S. Martinez. Distributed Control of Robotic Networks. A Mathematical Approach to Motion Coordination Algorithms. Princeton University Press, 2009.

[6] G. Ferrari-Trecate, L. Galbusera, M. Marciandi, and R. Scattolini. Model predictive control schemes for consensus in multi-agent systems with single- and double-integrator dynamics. IEEE Transactions on Automatic Control, 54(11):2560-2572, 2009.

[7] M. Fiacchini and I.-C. Morărescu. Set theory based condition in LMI form for network topology preservation for decentralized control. In Proc. of the 51st IEEE Conference on Decision and Control (CDC), pages 6229-6234, 2012.

[8] A. Jadbabaie, J. Lin, and A.S. Morse. Coordination of groups of mobile autonomous agents using nearest neighbor rules. IEEE Transactions on Automatic Control, 48:988-1001, 2003.

[9] S. Martin and A. Girard. Sufficent conditions for flocking via graph robustness analysis. In Proc. of the 49th IEEE Conference on Decision and Control, pages 6293-6298, 2010.

[10] R. Olfati-Saber and R.M. Murray. Consensus problems in networks of agents with switching topology and time-delays. IEEE Transactions on Automatic Control, 49:1520-1533, 2004.

[11] W. Ren and R.W. Beard. Consensus seeking in multiagent systems under dynamically changing interaction topologies. IEEE Transactions on Automatic Control, pages 655-661, 2005.

[12] R.T. Rockafellar. Convex Analysis. Princeton University Press, USA, 1970.

[13] M.M. Zavlanos and G.J. Pappas. Distributed connectivity control of mobile networks. IEEE Transactions on Robotics, 24(6):1416-1428, 2008 